\title{
Lepton flavour violation in the supersymmetric type-II seesaw mechanism
}

\author{
F. R. Joaquim* and Anna Rossi ${ }^{\dagger}$ \\ ${ }^{*}$ Departamento de Física Teórica \& Instituto de Física Teórica UAM/CSIC, Facultad de Ciencias C-XI, \\ Universidad Autónoma de Madrid, Cantoblanco, E-28049 Madrid, Spain \\ ${ }^{\dagger}$ Dipartimento di Fisica "G. Galilei”, Università di Padova I-35131 Padua, Italy
}

\begin{abstract}
We summarize the predictions for the radiative decays $\ell_{j} \rightarrow \ell_{i} \gamma$ within the context of the supersymmetric type II seesaw mechanism considering universal boundary conditions for the soft SUSY breaking terms. The dependence on the low-energy neutrino parameters is discussed and the deviations from the analytical results for large $\tan \beta$ analyzed.
\end{abstract}

Keywords: Supersymmetry, lepton flavour violation, neutrino physics PACS: $12.60 . J v, 14.60 . \mathrm{Pq}, 13.35 .-\mathrm{r}$

The observation of lepton flavour violation (LFV) signals (besides those manifest in neutrino oscillations) would be a clear and dramatic manifestation of new physics since, in the SM, they are strongly suppressed by the smallness of neutrino masses. A typical example where alternative LFV processes can be sizeable is the minimal supersymmetric standard model (MSSM) where they can be enhanced through one-loop exchange of sleptons (and gauginos) if their masses are not too far from the electroweak scale and do not conserve flavour.

Regarding flavour violation, most of the available studies rely on the most conservative scenario of universal sfermion masses at a high scale $\Lambda$. In such cases, flavour non-conservation in the sfermion masses might arise from renormalization group (RG) effects in the presence of flavour-violating Yukawa couplings [1]. In the so-called type I seesaw mechanism [2], these couplings are of the form $\mathbf{Y}_{N} N L H_{2}$ where $L$ is a lepton doublet, $\mathrm{H}_{2}$ the hypercharge $+1 / 2$ Higgs doublet of the MSSM and $N$ a heavy neutrino singlet which decouples at a scale $M_{N}$. From $\Lambda$ to $M_{N}$ the presence of $\mathbf{Y}_{N}$ induces LFV in the sfermion masses. On the other hand, the effective neutrino mass matrix after electroweak symmetry breaking reads $\mathbf{m}_{v}=v_{2}^{2} \mathbf{Y}_{N}^{T} \mathbf{M}_{N}^{-1} \mathbf{Y}_{N}$ where $\mathbf{M}_{N}$ is the heavy neutrino mass matrix. Therefore, one would expect to find a connection between low-energy neutrino parameters and the amount of flavour-violation induced in the slepton sector. This turns out to be impossible since one cannot reconstruct the high-energy neutrino parameters $\left(\mathbf{Y}_{N}\right.$ and $\left.\mathbf{M}_{N}\right)$ from low-energy measurements in the type I seesaw framework. One is therefore compelled to consider model-dependent conditions in order to make some predictions [3].

Let us now discuss what happens in the type II seesaw mechanism [4] where the MSSM particle is extended by adding a vector-like pair of heavy triplets which trans- form under the $S U(2)_{W} \times U(1)_{Y}$ gauge group as $T \sim$ $(3,1), \bar{T} \sim(3,-1)[5,[6]$. Besides the usual MSSM interactions one can write the following new terms in the superpotential]

$$
W_{T}=\frac{1}{\sqrt{2}} \mathbf{Y}_{T}^{i j} L_{i} T L_{j}+\frac{1}{\sqrt{2}} \lambda H_{2} \bar{T} H_{2}+M_{T} T \bar{T},
$$

where $i, j=e, \mu, \tau$ are family indices. $\mathbf{Y}_{T}^{i j}$ is a $3 \times 3$ symmetric matrix, $\lambda$ is a dimensionless unflavoured coupling and $M_{T}$ denotes the mass parameters of the triplets.

At the electroweak scale the Majorana neutrino mass matrix emerges and is given by

$$
\mathbf{m}_{v}^{i j}=\frac{v_{2}^{2} \lambda}{M_{T}} \mathbf{Y}_{T}^{i j}, \quad \mathbf{m}_{v}=\mathbf{U}^{\star} \operatorname{diag}\left(m_{1}, m_{2}, m_{3}\right) \mathbf{U}^{\dagger},
$$

where $m_{i}$ are the effective neutrino masses and $\mathbf{U}$ is the low-energy leptonic mixing matrix which can be written as

$$
\mathbf{U}=\mathbf{V} \cdot \operatorname{diag}\left(1, \mathrm{e}^{i \phi_{1}}, \mathrm{e}^{i \phi_{2}}\right), \mathbf{V} \equiv \mathbf{V}\left(\theta_{12}, \theta_{23}, \theta_{13}, \delta\right) .
$$

We have denoted the three mixing angles by $\theta_{12}, \theta_{23}$ and $\theta_{13}$, and the "Dirac" and "Majorana" CP-violating phases by $\delta$ and $\phi_{1,2}$, respectively. The matrix $\mathbf{V} \equiv$ $\mathbf{V}\left(\theta_{12}, \theta_{23}, \theta_{13}, \delta\right)$ can be parameterized as the CKM mixing matrix in terms of $\theta_{i j}$ and $\delta$. The neutrino masses can be expressed as:

$$
\begin{aligned}
\mathrm{NO}: m_{2}^{2} & =m_{1}^{2}+\Delta m_{\odot}^{2}, m_{3}^{2}=m_{1}^{2}+\Delta m_{\mathrm{atm}}^{2} \\
\mathrm{IO}: m_{2}^{2} & =m_{3}^{2}+\Delta m_{\mathrm{atm}}^{2}+\Delta m_{\odot}^{2}, m_{1}^{2}=m_{3}^{2}+\Delta m_{\mathrm{atm}}^{2},
\end{aligned}
$$

\footnotetext{
1 One can also include the term $\frac{1}{\sqrt{2}} \lambda_{1} H_{1} T H_{1}$ but this will be irrelevant for our discussion.
} 
where NO (IO) stands for considering a normal (inverted) order for the neutrino mass spectrum. The current allowed ranges for the mixing angles and neutrino mass-squared differences may be found in Ref. [7].

The presence of the Yukawa interactions $\mathbf{Y}_{T}$ may also induce LFV in the spleton mass matrix $\mathbf{m}_{\tilde{L}}$. This was first pointed out by Rossi in Ref. [6] in a framework where the soft sfermion masses are universal at a scale above $M_{T}$, i.e. $\mathbf{m}_{\tilde{L}}=m_{0}^{2} 1$.

Between the universality scale $\Lambda$ and $M_{T}$ the RG induced LFV matrix elements read [6]:

$$
\left(\mathbf{m}_{\tilde{L}}^{2}\right)_{i j} \simeq-\frac{9 m_{0}^{2}+3 a_{0}^{2}}{8 \pi^{2}}\left(\mathbf{Y}_{T}^{\dagger} \mathbf{Y}_{T}\right)_{i j} \ln \frac{\Lambda}{M_{T}},(i \neq j) .
$$

The most striking feature of this result is that the combination $\mathbf{Y}_{T}^{\dagger} \mathbf{Y}_{T}$ can be traded 2 by $\mathbf{m}_{v}^{\dagger} \mathbf{m}_{v}$ using Eq. (2) and therefore the dependence of $\left(\mathbf{m}_{\tilde{L}}^{2}\right)_{i j}$ on the low-energy neutrino parameters is now direct, contrarily to what happens in the type I seesaw mechanism. In particular, and taking into account Eqs. (2)-(4)

$$
\begin{aligned}
\mathrm{NO}:\left(\mathbf{m}_{v}^{\dagger} \mathbf{m}_{v}\right)_{i j} & =m_{1}^{2} \delta_{i j}+\Delta m_{\odot}^{2} \mathbf{V}_{i 2} \mathbf{V}_{j 2}^{*}+\Delta m_{\mathrm{atm}}^{2} \mathbf{V}_{i 3} \mathbf{V}_{j 3}^{*}, \\
\mathrm{IO}:\left(\mathbf{m}_{v}^{\dagger} \mathbf{m}_{v}\right)_{i j}= & m_{3}^{2} \delta_{i j}+\left(\Delta m_{\mathrm{atm}}^{2}+\Delta m_{\odot}^{2}\right) \mathbf{V}_{i 2} \mathbf{V}_{j 2}^{*} \\
& +\Delta m_{\mathrm{atm}}^{2} \mathbf{V}_{i 1} \mathbf{V}_{j 1}^{*},
\end{aligned}
$$

which reveals the fact that the LFV induced in the slepton masses does not depend on the lightest neutrino mass ( $m_{1}$ and $m_{3}$ for the normal and inverted mass spectrum, respectively) [8]. The existence of LFV in the slepton sector may lead to the enhancement of certain LFV processes which otherwise are very suppressed. Typical examples are the radiative decays $\ell_{j} \rightarrow \ell_{i} \gamma$ which can be naively estimated by

$\mathrm{BR}\left(\ell_{j} \rightarrow \ell_{i}+\gamma\right) \simeq \frac{\alpha^{3}}{G_{F}^{2}} \frac{\left|\left(\mathbf{m}_{\tilde{L}}^{2}\right)_{j i}\right|^{2}}{m_{S}^{8}} \tan ^{2} \beta \mathrm{BR}\left(\ell_{j} \rightarrow \ell_{i} v_{j} \bar{v}_{i}\right)$.

Here, $\alpha$ and $G_{F}$ denote the fine structure and Fermi constant, respectively, and $m_{S}$ a typical SUSY mass for the sparticles inside the loops. It is convenient to work with the quantities [6]

$$
\begin{aligned}
& R_{\tau \mu} \equiv \frac{\mathrm{BR}(\tau \rightarrow \mu \gamma)}{\mathrm{BR}(\mu \rightarrow e \gamma)} \simeq\left|\frac{\left(\mathbf{m}_{\tilde{L}}^{2}\right)_{\tau \mu}}{\left(\mathbf{m}_{\tilde{L}}^{2}\right)_{\mu e}}\right|^{2} \frac{\mathrm{BR}\left(\tau \rightarrow \mu v_{\tau} \bar{v}_{\mu}\right)}{\mathrm{BR}\left(\mu \rightarrow e v_{\mu} \bar{v}_{e}\right)}, \\
& R_{\tau e} \equiv \frac{\operatorname{BR}(\tau \rightarrow e \gamma)}{\operatorname{BR}(\mu \rightarrow e \gamma)} \simeq\left|\frac{\left(\mathbf{m}_{\tilde{L}}^{2}\right)_{\tau e}}{\left(\mathbf{m}_{\tilde{L}}^{2}\right)_{\mu e}}\right|^{2} \frac{\operatorname{BR}\left(\tau \rightarrow e v_{\tau} \bar{v}_{e}\right)}{\operatorname{BR}\left(\mu \rightarrow e v_{\mu} \bar{v}_{e}\right)},
\end{aligned}
$$

which do not depend (in most cases) on $\tan \beta$ and on the SUSY spectrum. In the above equations

\footnotetext{
2 Note that this is true only when the RG effects on the flavour structure of $\mathbf{Y}_{T}^{\dagger} \mathbf{Y}_{T}$ are negligible. See discussion at the end.
}
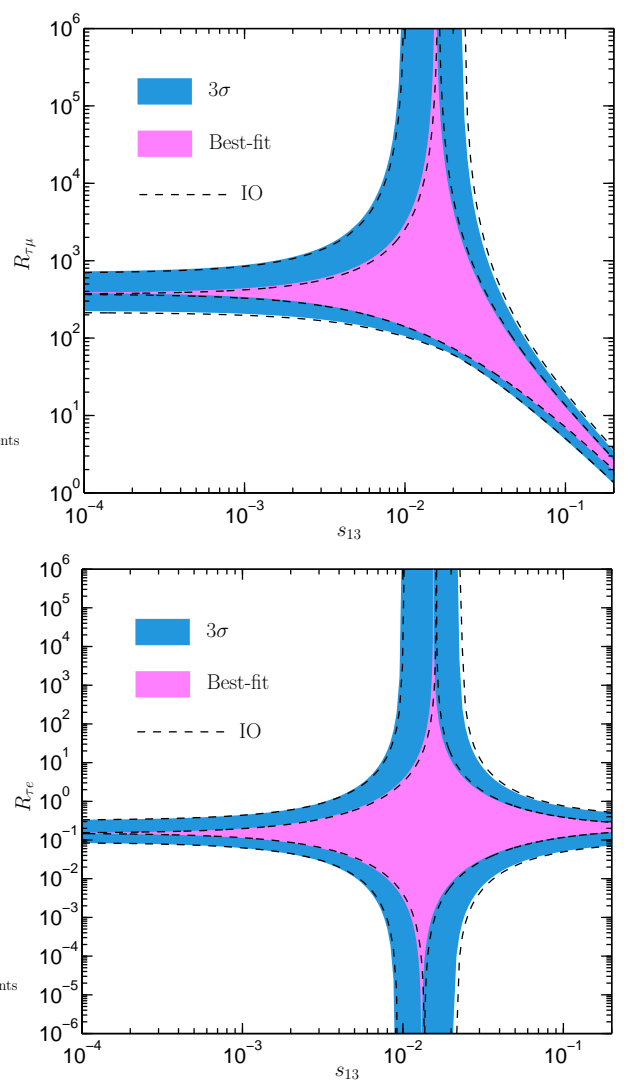

FIGURE 1. Allowed ranges for $R_{\tau \mu}$ (upper plot) and $R_{\tau e}$ (lower plot) as a function of $s_{13}$. The shaded regions correspond to the NO case and the dashed lines delimit the same regions for the IO spectrum.

$\operatorname{BR}\left(\tau \rightarrow \mu v_{\tau} \bar{v}_{\mu}\right) / \operatorname{BR}\left(\mu \rightarrow e v_{\mu} \bar{v}_{e}\right)=0.1737$ and $\operatorname{BR}\left(\tau \rightarrow e v_{\tau} \bar{v}_{e}\right) / \operatorname{BR}\left(\mu \rightarrow e v_{\mu} \bar{v}_{e}\right)=0.1784$.

Using now a standard parameterization for the elements of $\mathbf{V}$ we can write

$$
\begin{aligned}
& \left|\left[\mathbf{m}_{\tilde{L}}^{2}\right]_{\mu e}\right|^{2} \propto \frac{c_{13}^{2}}{4}\left[\rho^{2} c_{23}^{2} \sin ^{2}\left(2 \theta_{12}\right)+4 s_{13}^{2} s_{23}^{2}\right. \\
& \left. \pm 2 \rho s_{13} \cos \delta \sin \left(2 \theta_{12}\right) \sin \left(2 \theta_{23}\right)\right], \\
& \left|\left[\mathbf{m}_{\tilde{L}}^{2}\right]_{\tau e}\right|^{2} \propto \frac{c_{13}^{2}}{4}\left[\rho^{2} s_{23}^{2} \sin ^{2}\left(2 \theta_{12}\right)+4 s_{13}^{2} c_{23}^{2}\right. \\
& \left|\left[\mathbf{m}_{\tilde{L}}^{2}\right]_{\tau \mu}\right|^{2} \propto \frac{1}{4}\left\{\rho^{2} s_{13}^{2} \sin ^{2} \delta \sin ^{2}\left(2 \theta_{12}\right)\right. \\
& \left.+\left[c_{13}^{2} \sin \left(2 \theta_{23}\right) \mp \rho s_{13} \cos \delta \sin \left(2 \theta_{12}\right) \cos \left(2 \theta_{23}\right)\right]^{2}\right\} .
\end{aligned}
$$

and use this to express $R_{\tau \mu}$ and $R_{\tau e}$ in terms of the lowenergy neutrino parameters. We have used the notation $s_{i j} \equiv \sin \theta_{i j}, c_{i j} \equiv \cos \theta_{i j}$ and $\rho \equiv \Delta m_{\odot}^{2} / \Delta m_{\text {atm }}^{2}$. In the limit $s_{13} \rightarrow 0$ one gets $R_{\tau \mu} \simeq 0.7 s_{23}^{2} /\left[\rho^{2} \sin ^{2}\left(2 \theta_{12}\right)\right] \simeq$ 383.0 and $R_{\tau e} \simeq 0.18 \tan ^{2} \theta_{23} \simeq 0.2$. For $s_{13} \simeq 0.2$ one obtains $R_{\tau \mu} \simeq 0.17 c_{23}^{2} \cot ^{2} \theta_{13} \simeq 2.0$ and $R_{\tau e} \simeq$ 

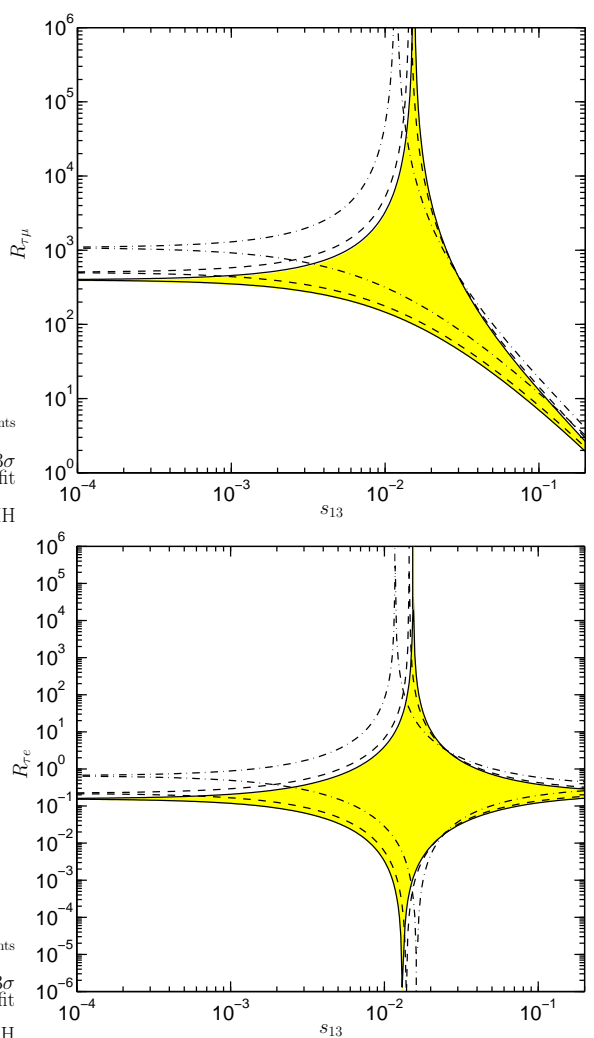

FIGURE 2. $\quad R_{\tau \mu}$ (upper plot) and $R_{\tau e}$ (lower plot) as a function of $s_{13}$. The shaded regions correspond to the analytical results using the best-fit values for the neutrino parameters and varying $\delta$ from 0 to $2 \pi$. The lines delimit the same region using the full numerical calculation of the processes and performing the running of all couplings and masses from low to high-energies and back. The solid, dashed and dash-dotted lines correspond to $\tan \beta=10,30,50$, respectively.

$0.18 \cot ^{2} \theta_{23} \simeq 0.2$. In Fig. 11 we present the allowed ranges for $R_{\tau \mu}$ and $R_{\tau e}$ considering the experimental allowed ranges for the neutrino parameters and varying the phase $\delta$ between 0 and $2 \pi$. The strong enhancements and suppressions observed in the plots for $\left|s_{13}\right| \simeq$ $\rho \cot \theta_{23} \sin \left(2 \theta_{12}\right) / 2 \simeq 0.016$ are due to cancelations in $\left|\left[\mathbf{m}_{\tilde{L}}^{2}\right]_{\mu e}\right|^{2}$ and/or $\left|\left[\mathbf{m}_{\tilde{L}}^{2}\right]_{\tau e}\right|^{2}$ for $\delta=0, \pi$ [see Eqs.(9) and Refs. [8]]. The above results have been obtained using the estimate given in Eq. 7 However, this estimate is valid in the limit of equal masses for the sparticles entering in the loop. Also notice that the above results have been obtained using the low-energy neutrino parameters. This approximation is valid if the RG effects in $\mathbf{Y}_{T}$ are negligible when one goes from low to high energies. This might not be the case if $\tan \beta$ is large. Moreover, for large $\tan \beta$, the left-handed staus get separated in mass from the smuon and selectron. This is due to running effects in the diagonal elements of the slepton mass matrix when the Yukawa of the $\tau$ is large. Putting all together, we ex- pect that the results for $R_{\tau \mu}$ and $R_{\tau e}$ deviate from the ones presented above when $\tan \beta$ is large. In Fig. 2] we present the results taking into account the best-fit values for the neutrino parameters. We see that as $\tan \beta$ increases, the results deviate from the ones given by the analytical expressions (which are shown in shaded). For instance, for $\tan \beta=50$ and small $s_{13}, R_{\tau \mu}$ is enhanced by a factor of approximately 2.5 with respect to the analytical result. This will be discussed in more detail in a forthcoming publication [9]. It is clear from Figs. 1 and 2 that if $s_{13}$ is close to the present upper bound (let's say $s_{13} \simeq 0.2$ ) then the present bound $\mathrm{BR}(\mu \rightarrow e \gamma)<1.2 \times 10^{-11} \mathrm{im}$ plies $\operatorname{BR}(\tau \rightarrow \mu \gamma) \lesssim 10^{-10}$ and $\operatorname{BR}(\tau \rightarrow e \gamma) \lesssim 10^{-11}$, which are both beyond future experiments. Therefore, if $s_{13}$ is large, the only decay which could be observed is $\mu \rightarrow e \gamma$.

\section{ACKNOWLEDGMENTS}

F. R. J. is supported by the EU 6th Framework Program MRTN-CT-2004-503369 "The Quest for Unification: Theory Confronts Experiment".

\section{REFERENCES}

1. F. Borzumati and A. Masiero, Phys. Rev. Lett. 57, 961 (1986).

2. P. Minkowski, Phys. Lett. B 67421 (1977); M. GellMann, P. Ramond and R. Slansky, in Supergravity, eds. P. Van Nieuwenhuizen and D. Freedman (North-Holland, Amsterdam, 1979),p. 315; T. Yanagida, in Proceedings of the Workshop on the Unified Theory and the Baryon Number in the Universe, eds. O. Sawada and A. Sugamoto (KEK, Tsukuba, 1979), p. 95; S.L. Glashow, in Quarks and Leptons, eds. M. Lévy et al., (Plenum, 1980, NewYork), p. 707; R.N. Mohapatra and G. Senjanović, Phys. Rev. Lett. 44, 912 (1980).

3. For a discussion on several examples see e.g., M. Raidal et al., arXiv:0801.1826 [hep-ph].

4. R. Barbieri, D.V. Nanopolous, G. Morchio and F. Strocchi, Phys. Lett. B 90, 91 (1980); R. E. Marshak and R. N. Mohapatra, VPI-HEP-80/02 Invited talk given at Orbis Scientiae, Coral Gables, Fla., Jan 14-17, 1980; T. P. Cheng and L. F. Li, Phys. Rev. D 22, 2860 (1980); M. Magg and Ch. Wetterich, Phys. Lett. B 94, 61 (1980); J. Schechter and J. W. F. Valle, Phys. Rev. D 22, 2227 (1980); R.N. Mohapatra and G. Senjanovic, Phys. Rev. D 23, 165 (1981); E. Ma and U. Sarkar, Phys. Rev. Lett. 80, 5716 (1998).

5. T. Hambye, E. Ma and U. Sarkar, Nucl. Phys. B 602 (2001) 23.

6. A. Rossi, Phys. Rev. D 66, 075003 (2002).

7. M. C. Gonzalez-Garcia and M. Maltoni, Phys. Rept. 460 (2008) 1 [arXiv:0704.1800 [hep-ph]].

8. F. R. Joaquim and A. Rossi, Phys. Rev. Lett. 97 (2006) 181801; Nucl. Phys. B 765 (2007) 71.

9. In preparation. 